\title{
The measurement of open apices of teeth to test chronological age of over 14-year olds in living subjects
}

\author{
Roberto Cameriere $^{\mathrm{a}, *}$, Hervoje Brkic ${ }^{\mathrm{b}}$, Branko Ermenc ${ }^{\mathrm{c}}$, Luigi Ferrante ${ }^{\mathrm{d}}$, \\ Maja Ovsenik $^{\mathrm{c}}$, Mariano Cingolani ${ }^{\mathrm{a}}$ \\ a Institute of Forensic Medicine, University of Macerata, Via Don Minzoni 9, 62100 Macerata, Italy \\ ${ }^{\mathrm{b}}$ School of Dental Medicine, Department of Dental Anthropology, University of Zagreb, Gunduliceva 5, HR-10000 Zagreb, Croatia \\ ${ }^{\mathrm{c}}$ Institute of Forensic Medicine, Faculty of Medicine, University of Ljubljana, Korytkova 2, 1000 Ljubljana, Slovenia \\ ${ }^{\mathrm{d}}$ Institute of Biochemical Biotechnologies, Polytechnic University of Marche, Ancona, via Ranieri 65, 60100 Ancona, Italy
}

Received 26 November 2006; received in revised form 16 March 2007; accepted 25 April 2007

\begin{abstract}
Age determination in living subjects is a problem of increasing interest in our community, due to the increasing numbers of individuals without identification papers, who have immigrated illegally or committed crimes, and for whom it is necessary to verify whether they have reached the age of 14 years in order to be charged legally.

Although the most widespread methods for age estimation refer to skeletal or dental analysis, these methods do present some drawbacks for identification of the age of 14 . The aim of the present study is to discriminate between children who are or are not 14 years of age or older by measuring the open apices of teeth.

We evaluated the OPGs of 447 persons aged between 12 and 16 years, of Italian, Croatian and Slovenian nationality. For each individual, dental maturity was estimated using the number of the seven left permanent mandibular teeth with root development complete, and normalized measurement of the open apices of the third molar.

The results revealed that an individual is considered to be 14 years of age or older if all seven left permanent mandibular teeth have closed apices and the normalized measurement of open apices of the third molar is lower than 1.1.
\end{abstract}

(C) 2007 Published by Elsevier Ireland Ltd.

Keywords: Forensic odontology; Age estimation; Third molar; Open apices of teeth

\section{Introduction}

The need to determine the age of living individuals is a problem of increasing interest in our community, due to the progressively higher number of persons not in possession of any document of identity or whose birth certificate may be suspected to be wrong, who have immigrated illegally or committed crimes, whose real age must be known in order to decide whether they can be charged, and whether they should be subjected to trial as of age or at least 14 years old. Also in cases of adoption, it is sometimes important to assess age when no birth certificate is available. In the last few years, therefore, forensic medicine has shown increasing interest in this problem

\footnotetext{
* Corresponding author. Tel.: +390733 2582625; fax: +3907332582635. E-mail address: r.cameriere@unimc.it (R. Cameriere).
}

[1-5] and in the reliability of methods for assessing biological age.

During the growth of a person, skeletal, odontological, anthropological and psychological methods allow an approximate assessment of age. Among the methods most frequently used for skeletal assessment are those concerning the left handwrist area [6] and FELS [7], which can produce estimates up to the age of 16 years, at which time wrist maturation is complete in $90 \%$ of subjects.

Numerous odontological studies have also been carried out to establish age, assessing mineralization within acceptable error limits.

The most common method for dental age assessment was first published by Demirjian et al. [8] and since then odontology has carried out numerous studies in this issue [9].

Nevertheless, to the best of our knowledge, few papers were addressed to assess if an individual is at least 14 years old. Since 
60

61 it is a cut-point more and more important in forensic sciences in 62 order to decide if a children can be charged legally, the purpose 63 of the present study was to examine the open apices of the teeth 64 in discriminating between children who are or are not 14 years 65 of age or older. If a child is younger than 12 or older than 16 66 years of age there are many different and reliable techniques to 67 assess that he/she is or is not younger than 14 years of age. For 68 instance, taking into account the results in [10,2], it is easy to assess for a children older than 16 years that he/she is older than 14 years of age. Furthermore, using the regression formula found in Cameriere et al. [11] it is possible to estimate that when all apices are closed a child is almost sure older than 12 years (the probability that a child is younger than 12 years is less than 1\%). Consequently, we considered children aged from 12 to 16 years old as our target population.

\section{Materials and methods}

\subsection{Subjects and materials}

Orthopantomograms (OPGs) of 447 persons aged between 12 and 16 years, of Italian, Croatian and Slovenian nationality, were evaluated (Tables 1 and 2).

Subdivision according to sex was similar, with $47 \%$ females and $53 \%$ males. Selection criteria for inclusion of OPGs in this study were: Caucasian origin; all teeth on the right lower jaw present; no obvious dental pathology on panoramic radiology related to the right lower jaw, tilted third molar. Only 21 (5.9\%) of the 447 OPGs examined, in which these criteria were not satisfied, were excluded.

To discriminate between individuals who are or are not aged 14 years or more, we analysed the apical ends of the roots of the seven left permanent mandibular teeth of each individual. Briefly, for each individual, we considered the following measures: (1) number of the seven left permanent mandibular teeth with root development complete, apical ends of roots completely closed $\left(N_{0}\right)$ and dichotomous variable $C$ (with $C=1$, if all seven left permanent teeth had completely closed apices; $C=0$, if at least one tooth had its apices not completely closed) and (2) third molar maturity index $\left(D_{3 \mathrm{M}}\right)$, i.e., sum of the distances between the inner sides of the two open apices when roots were developed; otherwise, $D_{3 \mathrm{M}}$ was obtained dividing crown length by tooth length of highest cusp [11].

Dental maturity was evaluated using the third molar maturity index $D_{3 \mathrm{M}}$ and the dichotomous variable $C$.

\subsection{Statistical analysis}

All measurements were carried out by two observers. In order to evaluate intra- and inter-observer reliability, the two observers made repeated measures of 30 OPGs at an interval of 2 weeks.

The intra- and inter-observer reproducibility of the sum of the distances between the inner sides of the two open apices divided by the tooth length $\left(D_{3 \mathrm{M}}\right)$ was studied using the concordance correlation coefficient, $\rho_{\mathrm{c}}$, and $\kappa$ statistics were used to measure the intra- and inter-observer reproducibility of the number of the seven right permanent mandibular teeth with root development complete $\left(N_{0}\right)$.

Using individual age as a dichotomous response variable $(F=1$ if an individual is at least 14 years of age, $F=0$ otherwise), and gender, nationality,

Table 1

Countries distribution of the sample

\begin{tabular}{lccc}
\hline Country & Females & Males & Total \\
\hline Italy & 84 & 85 & 169 \\
Croatia & 73 & 73 & 146 \\
Slovenia & 54 & 78 & 132 \\
Total & 211 & 236 & 447 \\
\hline
\end{tabular}

Table 2

Age and sex distribution of the sample

\begin{tabular}{lccr}
\hline Years & Females & Males & Total \\
\hline 12 & 43 & 66 & 109 \\
13 & 46 & 41 & 87 \\
14 & 53 & 50 & 103 \\
15 & 30 & 33 & 63 \\
16 & 40 & 45 & 85 \\
Total & 212 & 235 & 447 \\
\hline
\end{tabular}

$C$, and $D_{3 \mathrm{M}}$ as predictor variables, we derived a generalized linear model to predict whether an individual is older $(F=1)$ or younger $(F=0)$ than 14 years of age by using a logistic model as link function.

The predictive accuracy of the model was assessed by the determination of receiver operating characteristic curve (ROC curve).

All the significant variables were used to test the medico-legal question of whether an individual is older or younger than 14 years of age. The test was performed identifying a threshold (cut-off) that can be used to assign an individual to the population of the younger $(T=0)$ or older $(T=1)$ than 14 years of age.

Sensitivity $p_{1}$ of test (i.e., the proportion of children equal to or older than 14 years of age, which verifies event $T=1$ ) was evaluated, and also its specificity, $p_{2}$ (i.e., the proportion of individuals younger than 14 years of age that verify the event $T=0$ ).

Open apices in teeth may help in discriminating between children who are or are not aged 14 years or more, by using the post-test probability of being 14 years of age or more (i.e., the proportion of individuals aged 14 or over in whom event $F=1$ is verified). According to Bayes' theorem, post-test probability may be written as:

$$
p=\frac{p_{1} p_{0}}{p_{1} p_{0}+\left(1-p_{2}\right)\left(1-p_{0}\right)}
$$

where $p$ is post-test probability and $p_{0}$ is the probability that a child is equal to or older than 14 years, given that he/she is aged between 12 and 16 years, which represents our target population. This probability, $p_{0}$, was evaluated using the data obtained from the statistical offices of Slovenia, Croatia and Italy [12-14]. Since sensitivity and specificity, the determinants of post-test probability of being aged 14 years or more, were unknown probabilities, they were estimated using our sample subjects. Consequently, post-test probability $p$ in Eq. (2.1) became a sample statistic subjected to random error. Thus, confidence intervals were used to describe its uncertainty.

The expression of the asymptotic $(1-\alpha)$ per cent confidence interval for the post-test probability estimate may be written in terms of the estimates of adult subjects, sensitivity, specificity and their corresponding sample size, as follows:

$\left[\frac{1}{1+p \exp \left(z_{\alpha / 2} \sqrt{V}\right)}, \frac{1}{1+p \exp \left(-z_{\alpha / 2} \sqrt{V}\right)}\right]$,

with

$V=\frac{1-p_{1}}{n_{1} p_{1}}+\frac{p_{2}}{n_{2}\left(1-p_{2}\right)}$

where $n_{0}$ is the sample size, and $n_{1}$ and $n_{2}$ are the numbers of individuals who are or are not aged 14 years or more. Statistical analysis of data and related graphs was carried out with S-PLUS 6 statistical program (S-PLUS ${ }^{\circledR} 6.1$ for Windows Professional Edition Release 1) and the Microsoft Excel ${ }^{\circledR}$ program. The significance level was set at $5 \%$.

\section{Results}

For the number of the seven right permanent mandibular teeth with root development complete $\left(N_{0}\right)$, we did not observe any disagreement between two measurements made by the same observer, i.e., $\kappa=1$. 
Inter-observer reproducibility of $N_{0}$ was good with Cohen's $\kappa$ statistics $( \pm$ S.D.) at $\kappa=0.93 \pm 0.07$, indicating no significant inter-observer differences.

As regards the reproducibility of $D_{3 \mathrm{M}}$ measurements (sum of distances between inner sides of two open apices divided by tooth length), the estimated concordance correlation coefficient $( \pm$ S.D. $)$ was $\rho_{\mathrm{c}}=0.966 \pm 0.0005$ for observer $1, \rho_{\mathrm{c}}=0.964 \pm$ 0.0035 for observer 2 , and $\rho_{\mathrm{c}}=0.956 \pm 0.0076$, when the measures of both observers were compared.

Inter-observer reproducibility of $D_{3 \mathrm{M}}$ did not reveal significant intra- or inter-observer effects, indicating substantial homogeneity of evaluation between operators.

From the data at our disposal, it is inferred that, in $5.9 \%$ of the subjects examined, the third molar on the right lower jaw was not present.

For the remaining $94.1 \%$ of the data, we studied the extent to which the age of 14 years or more of an individual $(F=1)$ is related to the maturation degree of the third molar $\left(D_{3 \mathrm{M}}\right)$, the dichotomous variable $C$, gender ( 1 for male and 0 for female) and nationality of the children.

Let $p=P(F=1)$ the probability that the an individual is at least 14 years of age, we modeled the dependence of this probability on $D_{3 \mathrm{M}}, C$, gender and nationality using a linear logistic model:

$\operatorname{logit}(p)=b_{0}+b_{1}$ nationality $+b_{2}$ gender $+b_{3} C+b_{4} D_{3 \mathrm{M}}$.

To examine the effect of including one of the four factors in, or excluding it from the model, we considered the difference in deviance between two nested models (Table 3 ).

The change in deviance on adding the variables nationality and gender to a model that includes a constant term alone (null model) was not significant.

Instead, when $C$ or $D_{3 \mathrm{M}}$ were added to the null model, the deviance was reduced by highly significant amounts $(p<0.001)$.

In summary, the probability that an individual is aged 14 years or more depends both on the dichotomous variable $C$ which is related to the number of the seven left permanent mandibular teeth with root development complete and to the maturation degree of the third molar $D_{3 \mathrm{M}}$, but it does not significantly depend on gender and nationality. Hence Eq. (3.1) can be rewritten as:

$p=\frac{1}{1+\mathrm{e}^{-\left(b_{0}+b_{1} C+b_{2} D_{3 \mathrm{M}}\right)}}$.

Table 3

Deviance on fitting the considered linear models to the data

\begin{tabular}{lccclc}
\hline & d.f. & Dev. resid. & d.f. & Deviance & $p$ \\
\hline Null & - & - & 425 & 582.6 & - \\
Nationality & 1 & 1.6 & 424 & 581.0 & 0.20 \\
Gender & 1 & 0.1 & 423 & 580.9 & 0.71 \\
$C$ & 1 & 323.0 & 422 & 257.8 & $<0.001$ \\
$D_{3 \mathrm{M}}$ & 1 & 16.3 & 421 & 241.5 & $<0.001$ \\
\hline
\end{tabular}

Terms were added sequentially (first to last).
Table 4

Parameter estimates for logistic model (3.2)

\begin{tabular}{lclc}
\hline Parameter & Value & S.E. & $t$-Value \\
\hline Intercept & 0.308 & 0.530 & 0.581 \\
$C$ & 4.233 & 0.367 & 11.527 \\
$D_{3 \mathrm{M}}$ & -2.190 & 0.544 & -4.03 \\
\hline
\end{tabular}

The maximum likelihood estimates of the model parameters (Table 4) evaluated the probability that an individual was equal to or older than 14 years of age, $p$, given the values of the factor $C$ and covariate $D_{3 \mathrm{M}}$ through the logistic model (3.2).

The predictive accuracy of Eq. (3.2) and its discrimination capacity was also assessed by determining the ROC curve by classification matrices for different levels of predicted probability that an individual is of age. The resulting ROC curve (Fig. 1) has an area under the curve ( \pm S.D.) of $0.814 \pm$ 0.021 .

To test the legal question of whether an individual is older or younger than 14 years of age, a procedure had to be identified, such that an individual is assigned to the population of the younger than 14 years of age if the test is resulted negative $(T=0)$ and to the older population if the test is resulted positive $(T=1)$.

For forensic purposes, it is important that the test shows a low proportion of individuals younger than 14 years of age whose test is resulted positive $(T=1)$, and so it seemed appropriate to pay more attention to the chance of a false positive than to that of a false negative.

On these grounds, we established that an individual is considered equal or older than 14 years of age (the test is positive, $T=1)$ if $C=1$ and $D_{3 \mathrm{M}}$ is lower than $D_{3 \mathrm{M}}^{*}=1.1$; otherwise an individual is considered younger than 14 years of age (the test is negative, $T=0$ ).

The sensitivity of this test (the proportion of individuals being older or equal to 14 years of age whose test is positive) was $81 \%$, and its specificity (the proportion of individuals younger than 14 years of age whose test is negative) was $95 \%$.

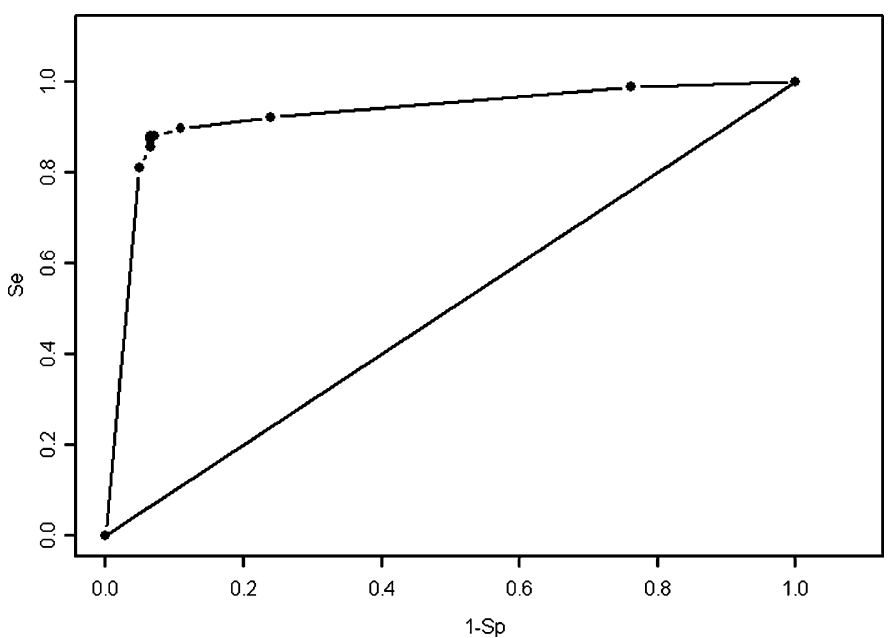

Fig. 1. Receiver operating characteristic curve for "14 years of age or older" status. 
Table 5

Classification table describing discrimination performance of the test

\begin{tabular}{lrrr}
\hline & \multicolumn{2}{c}{ Age } & \\
\cline { 2 - 4 } & $<14$ & $\geq 14$ & Total \\
\hline$T=0$ & 175 & 46 & 221 \\
$T=1$ & 9 & 196 & 205 \\
Total & 184 & 242 & 426 \\
\hline
\end{tabular}

Table 6

Distribution of the age for gender and test

\begin{tabular}{lllllllll}
\hline Gender & $T$ & Age & & & & & & \\
\cline { 3 - 8 } & & 12 & 13 & 14 & 15 & 16 & 17 & Total \\
\hline F & 0 & 0.21 & 0.18 & 0.04 & 0.03 & 0.03 & 0.00 & 0.47 \\
& 1 & 0.00 & 0.03 & 0.22 & 0.11 & 0.12 & 0.05 & 0.53 \\
\multirow{2}{*}{ Total F } & & 0.21 & 0.21 & 0.26 & 0.13 & 0.15 & 0.05 & 1.00 \\
M & 0 & 0.28 & 0.14 & 0.06 & 0.02 & 0.01 & 0.00 & 0.52 \\
& 1 & 0.00 & 0.02 & 0.15 & 0.13 & 0.14 & 0.04 & 0.48 \\
\multirow{2}{*}{ Total M } & & 0.28 & 0.16 & 0.21 & 0.15 & 0.15 & 0.04 & 1.00 \\
\hline
\end{tabular}

The proportion of individuals with correct classifications was $87 \%$ (Table 5).

In the sample, estimated post-test probability $p$ was 0.96 , with a $95 \%$ confidence interval, $\mathrm{CI}=(0.93,0.98)$. Hence, the probability that a subject positive on the test $(T=1)$ was equal or older than 14 years of age was $96 \%$. Consequently, the test yielded only $4 \%$ of false positives.

When subjects of 12 years of age were examined, using this test, no subjects were estimated as older than or equal to 14 years of age. In addition, when subjects of 13 years of age were examined, the test estimated only $2 \%$ of both males and females as older than 14 (Table 6). Furthermore, when subjects of 16 years of age were examined, none of them were estimated younger than 14 years of age.

\section{Discussion}

The need for effective and reliable scientific methods to determine age, particularly adult and over age of 14 years old, within a specific population has become increasingly important in resolving court cases. Since the methods usually applied for dental age estimation guarantee an error in estimated age of less than 2 years $[8,15,16]$, to estimate the post-test probability and prevalence of subjects older than 14 , we chose young people aged between 12 and 16 years old as an target population.

Our results showed that the test is not significantly dependent on the nationality (Croatian, Italian and Slovenian) of the children neither to their gender while it depends on the maturation degree of the teeth.

In this paper, our test estimates that a subject is older than 14 years of age if all the teeth, except the third molar, have closed apices (are fully grown) and the maturation degree of the third molar, $D_{3 \mathrm{M}}$, is equal or lesser than 1.1 .

When the suggested test was applied, the percentage of false negatives was $19 \%$ and the percentage of false positives was
$5 \%$. From a forensic point of view, the small percentage of false positives is particularly important, because it is a more serious error to consider a subject younger than 14 as chargeable than the error which does not consider a subject older than 14 as chargeable.

Our results confirmed that, if the root apices of the seven teeth in the right lower jaw of a child are completely closed, and the ratio of the sum of third molar root apices divided by tooth length is lower than 1.1, then there is a high probability that the subject is indeed at least 14 years of age. In fact, the estimated probability that a child with $C=1$ and $D_{3 \mathrm{M}} \leq 1.1$ has reached 14 years of age is $p=0.96$.

In Cameriere et al. [17] we analysed a technique to assess biological growth and age in children and adolescents using the wrist/hand area method. Ossification of the carpals showed good agreement with chronological age, and their mineralization lasts until the age of approximately 14. For this reason, analysis is in progress to assess the age of boys and girls in the 12-16 age bracket using a combination of carpal bone and tooth growth information.

\section{Acknowledgement}

This work was supported by a grant from the Polytechnic University of Marche.

\section{References}

[1] P.M. Garamendi, M.I. Landa, J. Ballesteros, M.A. Solano, Reliability of the methods applied to assess age minority in living subjects around 18 years old. A survey on a Moroccan origin population, Forensic Sci. Int. 154 (2005) 3-12.

[2] A. De Salvia, C. Calzetta, M. Orrico, D. De Leo, Third mandibular molar radiological development as an indicator of chronological age in a European population, Forensic Sci. Int. 146 (2004) S9-S12.

[3] N. Chaillet, A. Demirjian, Dental maturity in South France: a comparison between Demirjian's method and polynomial functions, J. Forensic Sci. 49 (2004) 1059-1066.

[4] A. Olze, A. Mahlow, S. Schmidt, K.D. Wernecke, G. Geserick, A. Schemling, Combined determination of selected radiological and morphological variables relevant for dental age estimation of young adults, Homo 56 (2005) 133-140.

[5] H.M. Liversidge, F. Lyons, M.P. Hector, The accuracy of three methods of age estimation using radiographic measurements of developing teeth, Forensic Sci. Int. 131 (2003) 22-29.

[6] J.M. Tanner, M.J.R. Healy, H. Goldstein, N. Cameron, Assessment of Skeletal Maturity and Prediction of Adult Height (TW3 Method), W.D. Saunders, London, 2001.

[7] A.F. Roche, W. Cameron Chumlea, D. Thissen, Assessing the Skeletal Maturity of the Hand-Wrist: Fels Method, Charles C. Thomas, Springfield, 1988.

[8] A. Demirjian, H. Goldstein, J.M. Tanner, A new system of dental age assessment, Hum. Biol. 45 (1973) 221-227.

[9] G. Willems, A review of the most commonly used dental age estimation techniques, J. Forensic Odontostomatol. 19 (2001) 9-17.

[10] H.H. Mincer, E.F. Harris, H.E. Berryman, The A.B.F.O. study of third molar development and its use as an estimator of chronological age, J. Forensic Sci. 3 (1993) 379-390.

[11] R. Cameriere, L. Ferrante, M. Cingolani, Age estimation in children by measurement of open apices in teeth, Int. J. Legal Med. 120 (2006) 4952 .

[12] Statistical Office of the Republic of Slovenia, http://www.stat.si/letopis/ index_letopis_en.asptable4,4. 
[13] Republic of Croatia central Bureau of Statistics, http://www.dzs.hr/ ljetopis/LjFrameE.htm.

[14] Istituto Nazionale di Statistica (ISTAT), http://demo.istat.it/pop2004/ index.html.

[15] C.S. Farah, D.R. Booth, S.C. Knott, Dental maturity of children in Perth, Western Australia, and its application in forensic age estimation, J. Clin. Forensic Med. 6 (1999) 14-18.
[16] A. Teivens, H. Mornstad, A comparison between dental maturity rate in 326 the Swedish and Korean populations using a modified Demirjian method, 327 J. Forensic Odontostomatol. 19 (2001) 31-35.

[17] R. Cameriere, L. Ferrante, D. Mirtella, M. Cingolani, Carpals and 329 epiphyses of radius and ulna as age indicators, Int. J. Legal Med. 7330 (2005) $1-4$. 